\title{
LA DERIVACIÓN VERBAL APRECIATIVA EN ESPAÑOL
}

\author{
ANTONIO RIFÓN SÁNCHEZ \\ (Universidad de Vigo)
}

\begin{abstract}
RESUMEN
This paper analyzes the verb formation by means of the called, in Spanish, affixal «apreciativos». The paper is split into three subparts that are occupied by others so many facets of this type of derivation. The first paragraph studies the nature of these affixes and, in it, we conclude that they have to be considered as suffixes; the second'is occupied by the study of the formal restrictions that limit the application of these suffixes to the verbs; and the mediator and the last analyzes the semantic features of the verbs that create this type of derivation.
\end{abstract}

\section{Afijos apreciativos: ¿Infijos o sufijos?}

Ambas posturas, la que los considera infijos y la que los considera sufijos, están presentes en los estudios morfológicos; la más común es la que considera a los afijos apreciativos como sufijos (Dardano (1978), Scalise (1984) o Corbin (1987)), aunque, también, hay autores que prefieren considerarlos como infijos. Varela Ortega $(1990,91)$, entre otros, los asigna a la morfología no concatenante y dentro de esta los considera infijos.

Esto explica por qué el infijo -it-, insertado dentro del segundo constituyente de la palabra, no puede cambiar la marca de género: no ve el siguiente ciclo que corresponde al nombre compuesto.

Como infijos también los ve Lang $(1990,167)$ :

An infixed element, usually corresponding to an emotive derivational morpheme, appears between the stem and the infinitive morpheme. 
Si situamos los afijos apreciativos dentro de una morfología concatenante, su caracterización como sufijos o infijos depende de la localización de las reglas apreciativas dentro del subcomponente morfológico.

Si consideramos que las reglas apreciativas se aplican con posterioridad a las flexivas, los afijos apreciativos deben ser tratados como infijos que se insertan entre el tema' y los sufijos flexivos; esta concepción supone que se han de solucionar ciertos problemas formales que surgen en la aplicación de dicha infijación. En la infijación verbal apreciativa se ha de explicar por qué se puede dar un cambio de la vocal temática de la base con respecto a la del derivado (dormir --> dormitar), por qué puede aparecer un sufijo verbal en el derivado inexistente en la base (picar --> picotear) y por qué puede desaparecer un sufijo verbal existente en la base (carrear --> carrejar). En la infijación nominal, nos enfrentamos a problemas semejantes como es la variación de la vocal del tema nominal de la base (perfum-e --> perfum-it-o-, jarab-e --> jarab-it-o- ${ }^{2}$, verd-e -$>$ verd-ec-it-o-) o la aparición en el derivado de una vocal del tema inexistente en la base (señal--> señal-it-a, pedal--> pedal-it-o-, añil--> añil-it-o-).

$\mathrm{Si}$, por el contrario, situamos la aplicación de las reglas apreciativas entre las reglas derivativas y las reglas flexivas, los afijos apreciativos han de ser sufijos que se unen a un tema - derivado o no- que sirve como base para crear otro tema al que se unen, posteriormente, los morfemas flexivos.

El proceso morfológico de la apreciación nominal es como sigue: el sufijo apreciativo se une a un tema - una base no flexionada - y crea un tema que, como todos, carece de marcas flexivas; ${ }^{3}$ la aparición de la marca flexiva en dicho tema se elige con respecto al paradigma flexivo al que pertenece el sufijo apreciativo adicionado y al género del nombre base.

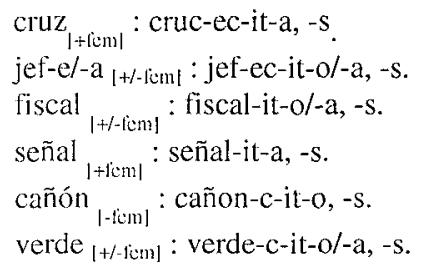

Tomamos tema como aquella unidad que resulta de eliminar de la palabra (flexiva) los morfemas flexivos. Esta concepción de tema se encuentra ya en Pena (1981).

Otra forma posible es jarab-e-c-it-o-que tiene dos explicaciones posibles: una alomorfia entre base y sufijo o conservación de la vocal del tema; aun considerando la -e- como la vocal del tema conservada, la existencia de un proceso de infijación conlleva problemas de interpretación.

Tomamos la marca flexiva nominal en sentido parecido a los words markers de Harris (1985). Los sufijos flexivos nominales no son realizados a no ser que se hallen en posición final de palabra y se sitúan sólo en esta posición cuando son insertados en la construcción sintáctica. Esta concepción de los procesos flexivos implica que, en el momento de unirse a las bases los sufijos apreciativos, los suffijos flexivos no han sido todavía realizados puesto que no están en posición fínal; los sufijos llexivos que se realizan en el derivado apreciativo no son, entonces, los del sustantivo base, sino los que corresponden a los morfemas apreciativos. 
vivaraz $_{\mid+/ \text {-lem! }}$ : vivarac-it-o/-a, $-\mathrm{s}$.

muchach-o/-a $[+$-feml $:$ muchachot-e/ -a, -s.

Vemos como en estos ejemplos se elige la marca dentro del paradigma al que pertenecen los sufijos $-i t-\mathrm{y}-$ ot $^{-}$

\begin{tabular}{|c|c|c|c|}
\hline & Masc & Fem & Masc Fen \\
\hline Sing. & $\mathrm{O}$ & A & E $A$ \\
\hline
\end{tabular}

$y$, dentro de cllos, se elige aquella marca de género que posee el nombre base: si este se femenino, se escoge la marca - $a$-; si es masculino, sólo la marca - 0 - o-ey si es masculino y femenino, tanto las marcas $-a$ - como $-o-0-e_{-}^{-4}$.

En la apreciación verbal, se ha de tener en cuenta que la vocal temática no es una marca de palabra, como bien indica Harris $(1985,39-40)$, ya que sus características son diferentes; la vocal temática se conserva a lo largo de las derivaciones, no así las marcas de palabra propuestas por Harris. En este momento, lo que nos interesa resaltar es que la vocal temática es asignada a cada tema verbal en el lexicón y, por tanto, tiene que ser tenida en cuenta en toda derivación, también en la derivación apreciativa. Los sufijos apreciativos verbales se comportan con respecto a la vocal temática de manera semejante a los temas verbales, la vocal temática les es asignada en el lexicón; en él, se les asigna a unos la vocal temática - $a$ - y a otros el sufijo verbal -e-(a)-por razones que trataremos al estudiar la semántica de estos sufijos 5 .

4 Los sufijos apreciativos, como podemos observar, regularizan la relación entre el género y número del sustantivo y su marca flexiva, correspondiéndose la marca de género masculino con la marea flexiva - $-o-o-e-$, el femenino con - a y el plural con $-s$. Esta regulación es un hecho normal en la creación de nuevas palabras, hecho ya apuntado por Harris (1985), quien establece tres tipos de paradigmas flexionales: el A-1 que corresponde a aquellos sustantivos que poseen género marcado y una marca diferente para cada género, o para el masculino y a para el femenino, A-2 que corresponde a aquellos que no tienen género marcado, pueden ser femeninos y masculinos y están acabados en - $e$ 0 consonante y el paradigma $B$ que contiene el resto de sustantivos. La creación de nuevas palabras se puede hacer sólo por medio de A-1 ० A-2, siendo A-1 el caso no marcado y el que más número de creaciones presenta:

Third, new words: Neologisms and borrowings are assigned to A-1 or A-2, never B, upon assimilation to the canonical morphological patterns of Spanish. Actually, this is a tautology: a potential new word can be considered 'really Spanish' only if it is assigned 10 one of the A classes.

In the secondary partitioning between $\mathrm{A}-1$ and $\mathrm{A}-2$, there is good reason to call $\mathrm{A}-1$ the unmarked case and A-2 the marked case. (Ibid., 43).

5 Se ha de indicar que los sufijos verbales son -ea-, -iza-, -ece-, -ifica-, -a-, - e- e - $i-$. Todos poseen su vocal temática puesto que sin ella no serían sufijos verbales. A pesar de la imposibilidad de separación de -e-, -iz-, -ific- y -ec- de su vocal temática -a-o-e-, preferimos grafiar estos sufijos como $-e-(a)-,-i z-(a)-,-i f i c-(a)-y-e c-(e)-$, indicando mediante los paréntesis y la separación la doble función 
Los problemas esbozados al tratar los afijos apreciativos verbales como infijos se solucionan si los consideramos como sufijos con las características arriba indicadas. El cambio de la vocal temática (dormir $->>$ dormitar) o la desaparición del sufijo verbal de la base en el derivado (carrear --> carrejar) se explican por medio del truncamiento de la marca verbal de la base, ya sea esta vocal temática, ya sufijo verbal.

En contra de este truncamiento tan general y obligatorio, se puede argumentar que los procesos de truncamiento no suelen ser nunca obligatorios ni se dan en todos los contextos en que los sufijos se aplican a una categoría; en su defensa, hemos de decir que este truncamiento es un caso especial, su carácter obligatorio y general se debe a que, de no producirse, se transgrede una regla morfológica básica del español que impide la aparición de dos marcas verbales, sean estas vocales temáticas o sufijos verbales, en ciclos morfológicos adyacentes. En español, no pueden aparecer combinadas de forma contigua marcas verbales $(-i-$ $a-,-e-(a)-a-,-i z-(a)-a-,-i z-(a)-e-(a)-,-i f i c-(a)-i z-(a)-, e t c$.$) ; este rasgo asemeja las$ marcas verbales a los sufijos flexivos.

En el caso de las derivaciones apreciativas, si atendemos únicamente a su estructura morfológica lineal, las marcas verbales no parecen estar en ciclos adyacentes:

$$
\text { bes- }(o)+\mathbf{a}+\mathrm{uc}+\mathbf{a}(\mathrm{r})
$$

Sí lo están si tenemos en cuenta su estructura morfológica real y, para establecer esta, hay que tener en cuenta dos hechos: los sufijos apreciativos son permeables a la categoría de la base y todo sufijo que cree verbos posee una marca verbal, sea esta vocal temática o sufijo verbal. Según estas características, la estructura morfológica de besuca( $r)$ no es:

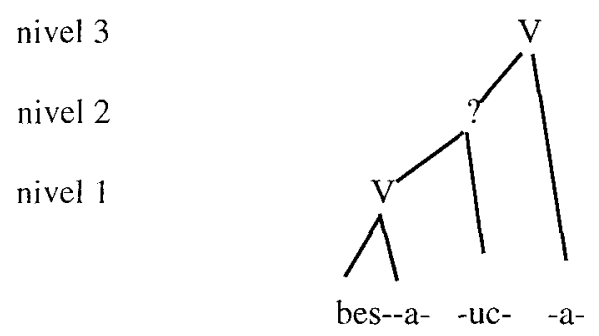

puesto que, o en el nivel 2 no existe ninguna etiqueta categorial, o, de ser esta verbo, el nivel 3 debería desaparecer al tener que unirse al mismo tiempo a la base el sufijo apreciativo - $u c$ - y la vocal temática - $a$-. La estructura morfológica de besucal $r$ sólo puede ser

de la vocal temática: vocal temática perteneciente al morfema derivativo y morfema flexivo marcador de la conjugación verbal. 


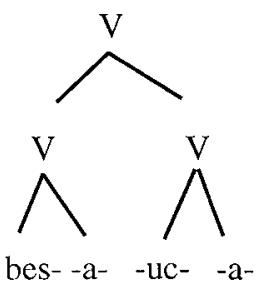

debido a que el sufijo - uc-, para crear verbos, necesita de la marca verbal.

La imposibilidad de aparición de dos sufijos verbales adyacentes es lo que provoca el truncamiento del sufijo verbal de la base. El hecho de que los sufijos apreciativos sean transparentes a la categoría de la base, pero no lo sean al morlema que marca dicha categoría se debe a que los sufijos apreciativos, al igual que toda raíz verbal, poseen marcas verbales propias asignadas en el lexicón, por lo que la marca de la base y del educto no tienen que coincidir.

En contra de este truncamiento tan general se manifiesta Corbin $(1987,127)$ :

-Première solution: supposer une troncation de l'affixe d'infinitif de la base, puis une suffixation globale de -oter, - -asser, -oyer, etc.

Cette solution présente un double inconvénient:

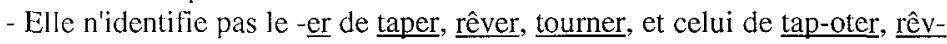
asser, tourn-over, ce qui est contredit par une analyse distributionnelle élémentaire:

tap-er $\rightarrow$ tap-ons

tapot-er $\rightarrow>$ tapot-ons

qui fait apparaitre l'autonomie de -ot- dans -ot-er.

- Elle implique la troncation de la partie finale de -oter, -asser, -oyer, etc. devant les suffixes dérivationnels et les affixes flexionnels, ce qui pose les problèmes déjà evoques, compliqués du fait qu'il s'agirait d'une troncation partielle et non totale du «suffixe» (ce n'est pas -oter qui serait tronqué, mais -er).

Nuestra propuesta salva ambos escollos. El sufijo apreciativo posee ya en el lexicón su propia marca verbal, sea esta la vocal temática - $a$ - o el sufijo verbal - $e$ (a)-, de manera que el sufijo apreciativo verbal no es -it-, sino-ita-o-itea-; en esta asociación entre marca verbal y sufijo apreciativo, la vocal temática no pierde su naturaleza de marcador de verbo, por lo que guarda relación con la vocal temática de la base; también puede sufrir truncamientos o alomorfias en los procesos flexivos, de la misma manera que en los procesos derivativos, truncamientos que no han de entenderse como truncamientos de una parte de un morlema, sino como truncamientos de la vocal temática. La asociación entre el sulijo apreciativo y la vocal temática se produce en el lexicón puesto que en él toda raíz o afijo perteneciente a la categoría verbo ha de poseer su vocal temática. 
A pesar de que se unan a la vez dos sufijos, el sufijo apreciativo y el sufijo verbal, a una base, esta afijación ha de ser interpretada como una única operación ya que ambos sufijos actúan como un todo indisoluble: la presencia del sufijo apreciativo verbal exige la presencia del sufijo - $e-(a)$ - o de la vocal temática - $a-$; al igual que ocurre con los sufijos verbales $-e-,-i z-,-i f i c$ - cuya presencia exige la de la vocal temática - $a$ - o al igual que ocurre con las raíces verbales, cuya presencia exige la de su vocal temática correspondiente.

Concluimos, por tanto, que los afijos apreciativos son sufijos, que el cambio de la vocal temática de la base con respecto al derivado y la posible desaparición del sufijo $-e-(a)$ - de la base se debe a un proceso de truncamiento, y que la aparición del sufijo -e-(a)- en el verbo derivado se debe a que los sufijos apreciativos necesitan de los morfemas verbales $-a-0-e-(a)$ - para poder crear verbos.

\section{Restricciones formales en las formaciones verbales apreciativas}

En primer lugar, hemos de reseñar que no existe un paralelismo total entre los afijos usados por la apreciación verbal y la nominal. Numerosos afijos utilizados en la apreciación nominal no lo son en la apreciación verbal (-on-, -at-, -uz-, -astr-, etc.) y, aunque menos, también existen afijos usados en la apreciación verbal que no lo son en la nominal (-ull-, -err-, -isc-, -urr-/-urri-). Los afijos utilizados en ambos tipos de formaciones tampoco coinciden en su productividad; afijos productivos en la derivación nominal no se presentan como productivos en la derivación verbal (-az-, -ill-, -it-, -ic-, etc.). Numéricamente, los afijos verbales apreciativos que podemos considerar más productivos son: -ot-, -et-, -aj-, -ej-, -orr-; los demás afijos, a la luz del número de verbos documentados, son menos

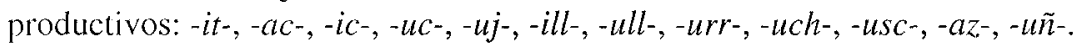

Ya se ha indicado que los sufijos apreciativos necesitan, para crear verbos, la presencia de marcas verbales, marcas que pueden ser la vocal temática - $a$ - o el sulijo verbal -e-(a)-. Nos centraremos, ahora, en el estudio de la combinación de los sufijos apreciativos y ambos sufijos verbales.

Algunos sufijos apreciativos toman como sufijo verbal -a-: -ac-(machacar), -uj-(apretujar, mamujar), -ull- (mamullar, mascullar), -uch-(escabuchar), -usc(churruscar), -az- (escobazar, aguazar), -uñ-(rascuñar, rasguñar). El número de verbos documentados creados por medio de estos sufijos es muy escaso, por lo que no podemos afirmar con plena seguridad que sea el sufijo - a- el único que tomen para formar verbos.

Otros sufijos toman como único sufijo verbal el sufijo -e-(a)-: -ot- (bailotear, chismotear, etc.), -orr- (andorrear, chismorrear, etc.). También el sufijo -ettoma como sulijo verbal único-e-(a)-(corretear, sopetear, etc.), aunque hemos documentado un caso con-a-(topar--> topetar). 
Hay otro grupo de sufijos (-it-, -ic-, - $\left.u c-,-a j_{-},-e j-,-u r r-,-i s c_{-}\right)$que pueden presentar tanto el sufijo - $e$-(a)- como la vocal temática - $a$ - y que, en muchos casos, crean sobre una misma base verbos con $-a-y$ con $-e-(a)-$ :

$$
\begin{array}{ll}
\text { bal-at-(r) } & -->\text { bal-it-a-(r)/bal-it-ea-(r) } \\
\text { bes-a-(r) } & -->\text { bes-uc-a-(r)/besuqu-ea-(r) } \\
\text { sob-a-(r) } & -->\text { sob-aj-a-(r)/sob-aj-ea-(r) } \\
\text { forz-a-(r) } & ->>\text { forc-ej-a-(r)/forc-ej-ea-(r) } \\
\text { cant-a-(r) } & -->\text { cant-urr-a-(r)/cant-urr-ea-(r) } \\
\text { com-c-(r) } & -->\text { com-isc-a-(r)/comisqu-ea-(r) }
\end{array}
$$

El escaso número de verbos con sufijación apreciativa documentados en el DRAE (1984) nos obliga a presentar la relación entre estos sufijos apreciativos y los sufijos verbales como tendencias sin elevarlas al rango de reglas.

La misma escasez de datos tampoco nos permite discernir si existe o no una tendencia clara a crear verbos apreciativos por medio de $-e-(a)$ - o por medio de $-a^{-}$, aunque en los datos documentados hay un predominio numérico de los verbos creados por medio de $-e-(a)$.

A pesar del escaso número de verbos documentados, podemos indicar que principalmente los verbos base pertenecen a la $1^{\text {a }}$ conjugación en $-a$-, aunque se toman también como bases verbos en $-e-(a)$ - y en $-e-\mathrm{e}-i$, pero se rechazan los verbos formados por medio de -iz-(a)-, -ec-(e)-e -ific-(a)-

La restricción formal que más interés ha suscitado en nuestro estudio señala que ciertos afijos apreciativos, diferenciados exclusivamente por la vocal inicial, se agrupan y se reparten el campo de aplicación a partir de la forma fonológica de la base:

$$
\begin{aligned}
& \text {-ot-/ -et-/ -it- } \\
& \text {-ac-/ -ic-/ -uc- } \\
& \text {-aj-/ -ej-/ -uj- } \\
& \text {-orr-/ -urr-/ -err- }
\end{aligned}
$$

Si la base presenta como vocal anterior al sufijo una vocal anterior (/e/, /i/), se clige como sufijo la variante que tenga como vocal inicial una vocal posterior (-ot-, -uc-, -uj-, -orr-, -urr-). Si la vocal de la base es una vocal postcrior $(/ \mathrm{o} /, / \mathrm{u} /)$, se elige como sufijo aquel que posea una vocal anterior (-et-, $-i t-,-i c-,-e j-,-e r r-)$. Los sufijos que presentan la vocal central $(-a c-,-a j-)$ pucden ser elegidos para adjuntarse a cualquier base con independencia de la naturaleza de la vocal de la base anterior al sufijo.

-ot-: bailotear, gimotear, fregotear, chismotear, picotear, etc.

-et-: corretear, golpetear, sopetear, topetar, toquetear, etc.

-it-: balitar, balitear, dormitar.

-ac-: machacar.

-ic-: aricar, lloriquear, mordicar. 
-uc-: besucar, besuquear, lambucear, machucar.

-aj-: borrajear, estirajar, quebrajar, sobajear, emplumajar, etc.

-ej-: bandejar, forcejar, voltejear, etc.

-uj-: apretujar, mamujar, mascujar, etc.

-orr-: andorrear, chismorrear, pintorrear, chinchorrear.

-err-: chuperretear.

-urr-: canturrar, canturrear.

A estos grupos de sufijos se les pueden dar varias interpretaciones. Se pueden tomar como sufijos que constan de una vocal más consonante (vT, vC, vRR, vJ), de manera que la realización de la vocal estaría determinada por la estructura fonológica de la base. Otro modo de entenderlos es considerar la existencia de un sufijo único para cada grupo y explicar el resto como variantes alomórficas debidas a un proceso fonológico de disimilación. Pero, si queremos mantener el paralelismo con la apreciación nominal, hemos de plantear una tercera vía en la que estos sufijos son, por lo menos algunos, sufijos diferentes agrupados únicamente en la apreciación verbal ya que, como sabemos, algunos de estos grupos de sufijos se presentan en la apreciación nominal como claramente diferentes: uno aumentativo (-ot-) y otro diminutivo $(-e t-)^{6}$.

\section{Características semánticas de las reglas verbales apreciativas}

Este apartado lo dedicaremos al estudio semántico de los sufijos verbales apreciativos e intentaremos establecer los paralelismos existentes entre apreciación verbal y nominal.

A la apreciación nominal se le ha reconocido tradicionalmente la expresión de dos valores semánticos: un valor denotativo, en el que se encuadran los valores diminutivo y aumentativo, y otro connotativo, en el que están incluidos como valores principales el positivo y el negativo; así, Dardano $(1978,96)$ indica:

Secondo una prospettiva tradizionale, la differenziazione operata dagli alterati è carattere dimensionale e nozionale: da una parte c'è un valore diminutivo/accrescitivo, dall'altra un valore positivo/ negativo.

El propio Dardano $(1978,105)$ añade a los sufijos verbales apreciativos el valor aspectual frecuentativo:

Questa transformazione produce frequentativi, diminutivi e accrescitivi.

La combinación de sufijos apreciativos también presenta fuertes restricciones; sólo los suffijos -arr-, err- y -orr- pueden combinarse con el resto y, además, son escasísimos los casos documentados: pintarrajar, chuperretear, beborrotear, cantarrinar. 
Creemos que ambas consideraciones son válidas, pero que se han de reestructurar para tener una visión correcta de la organización de los valores semánticos en los sufijos apreciativos. Pena $(1993,274)$ reconoce la existencia en la derivación verbal apreciativa de dos dimensiones:

la cuantificadora o numérica y la valorativa, y probablemente alguna más no fácil de identificar de momento.

Estas dos dimensiones pueden ser, como apunta el propio Pena, agrupadas en dos: la dimensión aspectual y la nocional.

En la dimensión aspectual, el significado expresado por la apreciación verbal se refiere siempre a la dimensión aspectual número entendida tal como lo hace Coseriu $(1980,21)$ :

4.2.2. «Nombre verbal» ou itération. C'est la dimension correspondant à des oppositions que sémelfactif-répété (itératif ou frécuantatif). Cf. russe govorit', «parler»,-govarivat' «(à plusieurs reprises)», ou bien, dans les langues romanes, les oppositions lexicales qu'on a dans le cas des «diminutifs» verbaux (fr. sauter-sautiller, esp. besar-besuquear).

Como indica Pena $(1993,274)$, la dimensión aspectual número posee dos oposiciones que pueden ser expresadas por las reglas apreciativas. Una oposición la constituye la expresión de la iteratividad/habitualidad frente a la semelfactividad y otra el grado de intensidad de la realización de la acción en la que podemos distinguir el grado atenuativo, el grado normal de intensidad y el grado intensivo; ambas oposiciones aspectuales, aunque remiten a la dimensión número, presentan características diferentes y se refieren a aspectos distintos de dicha dimensión.

En la primera oposición, se indica la cantidad numérica de acciones idénticas que se realizan: si la acción se realiza una sola vez, esta es semelfactiva; si se realiza más de una vez en una misma ocasión, es iterativa y, si se realiza más de una vez pero en ocasiones diferentes, es habitual.

La segunda oposición aspectual se refiere al grado de intensidad con el que se realiza una acción y está estructurada en un grado normal o no marcado, expresado por el verbo base, y los grados intensivo y atenuativo, expresados por los verbos derivados a través de los sufijos apreciativos. El grado intensivo expresa que, en la acción, el Agente ha puesto mayor grado de intensidad de la necesaria para realizarla; el grado atenuativo implica que el Agente ha puesto menor intensidad de la necesaria para realizar la acción de una forma normal.

Estas dos dimensiones aspectuales pueden relacionarse con dimensiones nominales. La dimensión aspectual que indica el número de repeticiones y que opone el semelfactivo al iterativo y habitual es el correlato verbal del número nominal ya que ambas dimensiones expresan la cantidad numérica de referentes 
iguales. En los verbos, el referente es una acción y la dimensión aspectual de número indica, por tanto, pluralidad de acciones idénticas; en los sustantivos, el referente es una clase y la dimensión aspectual indica la pluralidad de objetos de una misma clase. La dimensión aspectual de intensidad de la acción se puede comparar con la dimensión nominal que opone el diminutivo y el aumentativo; en la dimensión aspectual verbal lo que aumenta o disminuye es la intensidad con que se realiza la acción, mientras que en la nominal lo que aumenta o disminuye es el tamaño u otros rasgos del objeto.

Además de las dos dimensiones aspectuales, en la sufijación verbal apreciativa entra en juego una tercera dimensión: la dimensión nocional. Esta dimensión añade un valor subjetivo de tipo connotativo, por medio del cual, el hablante puede ver la acción desde un punto de vista positivo o negativo; como podemos observar esta dimensión también entra en juego en la apreciación nominal.

De la misma manera que en la apreciación nominal a los valores aumentativo y diminutivo se les pueden añadir los valores nocionales positivo y negativo o cstos presentarse de forma independiente; en la apreciación verbal a los valores aspectuales intensivo y atenuativo se les pueden añadir connotaciones positivas o negativas, aunque, en el caso de los verbos, el único valor que casi con exclusividad se presenta es el negativo (mascujar «mascar mal», bailotear «bailar mucho y sin gracia»).

Volvemos ahora sobre la dimensión aspectual número que opone la semelfactividad a la iteratividad y habitualidad. La sufijación verbal apreciativa crea tanto verbos semelfactivos como verbos iterativos o habituales por medio de los sufijos verbales - $a-$ y $-e-(a)$-; esta capacidad formativa ha de ser comparada con la capacidad formativa de ambos sufijos en la derivación verbal no apreciativa. La formación de verbos en español elige como sufijos creadores de verbos iterativos o habituales a los sufijos $-a$ - y $-e-(a)$-; el sufijo $-e-(a)$ - es el miembro marcado de ambos sufijos y crea verbos con significado iterativo o habitual; el sufijo - $a$ - es el miembro no marcado por lo que puede crear verbos tanto con significado iterativo o habitual como con significado semelfactivo.

En la derivación verbal apreciativa se siguen las mismas pautas de creación que en la derivación verbal no apreciativa. Los verbos creados por medio de -e(a)expresan iteratividad o habitualidad?

Beborrotear «beber a menudo y en poca cantidad»; chismotear «traer y llevar chismes»;

Utilizamos las definiciones de los verbos documentadas en el Diccionario de la Real Academia (DRAE) (1984) y en el Diccionario de Uso del Español de M. Moliner (1970) designado por las siglas (DUE). En caso de que al lado del significado del verbo no aparezca ninguna de estas siglas se trata de un significado documentado en el DRAE, diccionario que ha sido usado como básico al lo largo de lodo el estudio. 
picotear «golpear o herir las aves con el pico» $(D R A E) /$ (1) «picar algo las aves», (2) «coger trocitos de algo para comerlos o ir tomando de cuando en cuando una cosa de comer en poca cantidad» (DUE);

toquetear «locar reiteradamente y sin tino ni orden» $(D R A E) /$ «tocar una cosa reiteradamente y con pesadez» $(D U E)$;

traquetcar «hacer ruido estruendo o estrépito» (DRAE)/ «moverse reiteradamente una cosa produciendo ruido» $(D U E)$;

chupetear «chupar poco y con frecuencia» $(D R A E) /$ «chupar algo con insistencia» $(D U E)$;

gemiquear «gemir repetidamente»;

borrajear «hacer rúbricas, rasgos o figuras por mero entretenimiento o por ejercitar la pluma»;

callejear «andar frecuentemente y sin necesidad de calle en calle»;

andorrear «andar de una parte a otra, afectando diligencia, sin hacer cosa de sustancia» (DRAE)/ «ir de un lado para otro» (DUE);

chinchorrear «traer y llevar chismes y cuentos».

Incluso, hay verbos apreciativos creados por medio de -e-(a)- en los que, si bien su acción no es necesariamente repetida, al ser derivados por -e-(a)- dicha acción es vista como realizada en fases idénticas que se suceden:

Bailotear «bailar mucho y en especial cuando se hace sin gracia ni lormalidad» (DRAE);

gimotear «gemir ridículamente sin bastante causa»;

Iloriquear «llorar sin fuerza y $\sin$ bastante causa» (DRAE)/ «llorar débilmente como sin ganas y con un lloro monótono» (DUE).

Por medio del sufijo - $a$ - se crean verbos que expresan iteratividad o habitualidad

Mamujar «mamar como sin gana, dejando el pecho y volviéndolo a tomar»; mamullar «comer o mascar con los mismos ademanes y gestos que hace el que mama».

Machacar «golpear una cosa para quebrantarla o deformarla»;

bandejar «sostener bandos»,

y, también, verbos que expresan semelfactividad:

Aricar «arar superficialmente»; cnamorisacar «enamorarse superficialmente».

Esta doble posibilidad expresiva del sufijo $-a$ - (expresar tanto semelfactividad como itcratividad o habitualidad) es la que permite que se creen verbos en -e-(a)y en - cl- sinónimos con significado iterativo o habitual:

Balitar «balar con frecuencia»; 
balitear «balitar».

Besucar «besuquear»;

besuquear «besar repetidamente».

Comiscar «comer a menudo de varias cosas en pequeñas cantidades»; comisquear «comiscar».

Mordiscar «picar o punzar como mordiendo/ quitar poco a poco cosas muy pequeñas»;

mordisquear «picar o punzar como mordiendo».

El hecho de que la expresión de la semelfactividad, iteratividad y habitualidad sigan las mismas pautas en la derivación verbal no apreciativa y en la apreciativa nos lleva a pensar que, en esta última, la expresión de dichos valores no debe ser achacada a los sulijos apreciativos, sino a los verbales con los que se combinan; esto no quiere decir que la apreciación y la expresión del número aspectual (semellactividad, iteratividad y habitualidad) sean totalmente independientes, parece que existe una cierta solidaridad entre ambas, lo que ocurre es que los sufijos apreciativos no tienen capacidad para expresar dicha dimensión y son los morfemas verbales los encargados de dicha tarea.

Asignar la expresión de la dimensión aspectual número a los morfemas verbales permite establecer dos principios que consideramos fundamentales. En primer lugar, permite mantener la identidad de los morfemas verbales - $a$ y $-e-(a)$ - independientemente de que se presenten en la derivación verbal no apreciativa o en la derivación verbal apreciativa. En segundo lugar, nos permite guardar la identidad entre los sufijos apreciativos nominales y los verbales, al eliminar de los sufijos apreciativos verbales significados extraños a los sulijos apreciativos nominales.

Teniendo en cuenta lo dicho, podemos concluir que los sufijos apreciativos nominales y verbales aportan al derivado dos significados: un significado aspectual intensivo (diminutivo/aumentativo, intensivo/atenuativo) y un significado connotativo (positivo/negativo). Podemos concluir, además, que la dimensión aspectual número es expresada en la apreciación nominal por los sulijos de número y en la apreciación verbal por los sufijos verbales $-a$ - y -e-(a)-; como podemos observar, nuestra propuesta mantiene la identidad y semejanza entrc los morfemas apreciativos verbales y nominales.

Tradicionalmente se dividen los sufijos apreciativos dependiendo de la especialización significativa de cada uno de ellos: sufijos aumentativos (-az-,-ón, -ot-), diminutivos (-at-, -et-, -it-, -ill-, etc.) y peyorativos (-ac-, -uc-, -ach-, -uch-, $-u j_{-},-u z_{-}$, etc.). Los significados aumentativo y diminutivo no excluyen la existencia de contenidos connotativos afectivos (connotación positiva o negativa), connotaciones que se derivan de la propia interpretación que se puede realizar de los significados aumentativo y diminutivo: 
Il carattere dimensionale e quello nozionale non si escludono, anzi si richiamano a vicenda: alla piccoleza si riferisce la delicatezza e la gentilezza oppurc la debolezza e la meschinità; alla grandeza si referisce la forza oppure la cattiveria e la brutteza. (Dardano 1978, 96).

En la apreciación verbal tal divisón se difumina y no se establece una relación biunívoca entre los sufijos y los significados intensivo y atenuativo; a un sufijo caracterizado tradicionalmente como aumentativo le correspondería el significado intensivo, pero no siempre ocurre así: el sufijo -ot-, por ejemplo, caracterizado como aumentativo, puede expresar tanto intensión como atenuación 8 .

Verbos intensivos:

Bailotear «bailar mucho»;

parlotear «hablar mucho»;

pisotear «pisar algo violentamente» $(D U E)$.

Verbos atenuativos:

Beborrotear «beber a menudo y en poca cantidad»;

picotear «coger trocitos de algo para comerlos o ir tomando de cuando en cuando una cosa para comer en pequeña cantidad» (DUE).

Lo mismo ocurre en el sufijo - et- caracterizado como diminutivo:

Verbos intensivos:

Clavetear «clavar clavos desordenadamente o más de los necesarios en algún sitio» (DUE);

traquetear «hacer ruido, estruendo, estrépito».

Verbos atenuativos:

Corretear «correr en varias direcciones dentro de limitado espacio» (DUE).

Los sulijos apreciativos etiquetados como peyorativos pueden no sólo crear verbos con dicha nota connotativa, sino también con significado intensivo o atenuativo:

Verbos intensivos:

Apretujar «apretar mucho o reiteradamente»;

sobajar «manosear una cosa con fuerza ajándola»;

lamiscar «lamer de prisa y con ansia».

Verbos atenuativos:

Mamujar «mamar como sin gana»;

canturrear «cantar a media voz»;

aricar" «arar superficialmente».

8 Subrayamos aquellas palabras de la paráfrasis que pueden señalarnos de alguna manera el carricter atenuado o intensivo del verbo. 
En algunos casos, las definiciones de los diccionarios nos indican que el verbo creado puede tener tanto significado intensivo como atenuativo:

\author{
Significados intensivos: \\ Chupetear «chupar algo con insistencia» (DUE); \\ golpetear «golpear viva y continuamente» $(D R A E)$. \\ Significados atenuativos: \\ Chupetear «chupar poco y con frecuencia» (DRAE); \\ golpetear «dar golpes poco violentos seguidos» (DUE).
}

No podemos, por tanto, hablar de la existencia de sufijos apreciativos verbales aumentativos (intensivos), ni diminutivos (atenuativos), ni peyorativos; todo sufijo puede expresar una u otra opción. El hecho de que dichos sufijos no tengan una especialización significativa es lo que facilita que estos se agrupen por su afinidad en cuanto al significante y se combinen para crear verbos sólo con ciertas bases dependiendo del contexto fonológico de las mismas, como hemos visto al tratar las restricciones formales de combinación.

En cuanto a las notas nocionales aportadas por los sufijos apreciativos parece que no existe una oposición de tipo positivo/negativo al igual que en los nombres; en los verbos, esta oposición se da entre una ausencia de nota connotativa, es decir, verbos que no expresan ninguna nota connotativa y verbos con una connotación peyorativa; tampoco, al contrario de lo que ocurre en los nombres, existen unos sufijos especializados en la expresión de la peyoratividad. Los tradicionalmente llamados sufijos peyorativos pueden expresar tanto connotación peyorativa como no añadir ninguna connotación a la acción verbal:

Verbos con connotación peyorativa:

Mamujar «mascar mal o con dificultad»;

andorrear «andar de una parte a otra, afectando diligencia, sin hacer cosa de sustancia»;

lloriquear «llorar sin fuerza y sin bastante causa»;

pintorrear «manchar de varios colores y sin arte una cosa».

Verbos sin connotación peyorativa:

Olisquear «oler con cuidado o persistencia»;

estirajar «estirar una cosa deformándola»; aricar «arar superficialmente».

De la misma manera, los sufijos aumentativos y diminutivos pueden añadir al verbo una cierta connotación peyorativa:

Parlotear «hablar mucho y sin sustancia»;

toquetear «tocar una cosa reiteradamente y sin tino ni orden»;

lavotear «lavar aprisa mucho y mal». 
Crecmos que la expresión de la peyoratividad en los verbos es más una cuestión de discurso que una nota expresada realmente por los sufijos apreciativos; estos sufijos sólo capacitan al verbo para expresar dicha connotación, pero la expresión de esta se ha de apoyar además en otros recursos lingüísticos (la entonación, por ejemplo) y extralingüísticos, aunque hemos de reconocer que algunos de estos verbos parecen portar dicha connotación per se, sin necesidad de ningún apoyo exterior (mamullar «comer como quien mama», lavotear «lavar de prisa mucho y mal»).

Hcmos visto, pues, que la derivación verbal apreciativa se encuadra en tres oposiciones significativas: un significado aspectual expresado por los sufijos verbales de la formación y que se estructura en la oposición iterativo o habitual frente a semelfactivo, un significado aspectual indicado por los propios sufijos apreciativos que indica la intensidad con la que se realiza la acción y que se agrupa en la oposición de los significados intensivo o atenuativo frente al grado normal de intensidad y un tercer rasgo de carácter connotativo peyorativo que en muchos casos necesita ser reforzado por medio de otros recursos además de la aparición del sufijo apreciativo.

\section{Referencias bibliográficas}

Corbin, Daniclle (1987): Morphologie derivationnelle et structuration du lexique. Tübingen, Max Niemeyer.

Coseriu, E. (1980): «Aspect verbal ou aspects verbaux? Quelques questions de théorie el de methode» en J. David y R. Martin (eds.) La notion d'aspect. París, Klincksieck, Págs. 13-27.

Dardano, M. (1978): La formazione delle parole nell'italiano di oggi. Roma, Bulzoni.

Harris, James W. (1985): «Spanish word markers» en F. H. Nuessel (ed.) Current issues in hispanic phonology and morphology. Bloomington, IULC, págs. 3454.

Lang, Mervyn F. (1990): Spanish word formation: productive derivational morphology in the modern lexis. Nueva York, Croom Helm.

Lázaro Mora, Fernando (1977): «Morfología de los sufijos diminutivos -ito(a), -ico(a), -illo(a)»Verba, 4, págs. 115-125.

Lázaro Mora, Fernando (1981): «Los derivados sustantivos con -ete/-eta» Boletín de la Real Academia Española, 61, págs. 481-496.

Lázaro Mora, Fernando (1993): «Compatibilidad entre lexemas nominales y sufijos diminutivos» en Soledad Varela Ortega (ed.) La formación de palabras. Madrid, Síntesis, págs. 303-315.

Pena, Jcsús (1991): «La palabra: estructura y procesos morfológicos» Verba, 18, págs. 69-128.

Pena, Jesús (1993): «La formación de verbos en español» en Soledad Varela Ortcga (ed.) La formación de palabras. Madrid, Síntesis, págs. 217-281. 
Scalise, Sergio (1984): Generative morphology. Dordrecht, Foris.

Varela Ortega, Soledad (1990): Fundamentos de morfología. Madrid, Síntesis. 